\title{
Influência da Limitação da Amplitude de Movimento Sobre a Melhora da Flexibilidade do Ombro Após um Treino de Seis Semanas
}

\author{
Influence of the Range of Motion (Rom) Limitation on the Shoulder \\ Flexibility Improvement After a Six-Week Training
}

Daniel Câmara Azevedo

Silvia Coda de Carvalho

Elisa Westin Prado Soares Leal'

Sara Peres Damasceno 1

Manuela Loureiro Ferreira

1. Pontifícia Universidade Católica de Minas Gerais- PUC-Minas - Belo Horizonte - Minas Gerais

Endereço para correspondência: Departamento de Fisioterapia - ICBS Av Dom José Gaspar, 500 Coração Eucarístico - Belo Horizonte - Minas Gerais - CEP 30535-610

Email: danielazevedo@pucminas.br

Submetido em 04/03/2006 Versão final recebida em 22/09/2006 Aceito em 02/10/2007

\begin{abstract}
RESUMO
Estudos anteriores têm mostrado que o nível aeróbico e de força pré-treinamento influenciam os ganhos obtidos após um período de treino. Nenhum estudo investigou esta relação em um programa de flexibilidade. O objetivo deste estudo foi observar a influência da limitação da amplitude de movimento (ADM) sobre a melhora da ADM de rotação externa de ombro após um programa de alongamento de seis semanas. 30 voluntários, estudantes de fisioterapia, com limitação da ADM de rotação externa de ombro foram divididos de forma randomizadas em três grupos: grupo controle (GC), grupo experimental 1 (GE1), com maior limitação de ADM e grupo experimental 2 (GE2), com menor limitação de ADM. Os sujeitos do grupo experimental foram submetidos a um programa de seis semanas de alongamento ativo para melhora da ADM de rotação externa de ombro. Na análise dos resultados, o teste de ANOVA one-way mostrou uma diferença significativa entre a média do ganho de ADM de rotação externa de ombro entre os três grupos $(p=0,001)$, sendo que o GE1 teve o maior ganho de $\operatorname{ADM}\left(30,1^{\circ} \pm 8,6^{\circ}\right)$ seguido pelos grupos GE2 $\left(15,2^{\circ} \pm 7,5^{\circ}\right)$ e $\mathrm{GC}\left(1,1^{\circ} \pm 5,8^{\circ}\right)$. O teste de correlação de Pearson mostrou uma correlação negativa significativa entre ADM prévia e ganho de $\operatorname{ADM}(r=-0,70, p=0,001)$. Os resultados deste estudo mostraram que a ADM prévia de rotação externa de ombro influencia o ganho de ADM deste movimento após um programa de alongamento de seis semanas em uma população jovem e saudável. Indivíduos com maior limitação de ADM respondem com um ganho de ADM mais pronunciado.
\end{abstract}

Palavras-chave: músculo, reabilitação, treinamento

\begin{abstract}
Previous research has shown that pre-training aerobic and strength status can influence on the training results, with untrained individuals presenting a more pronounced improvement. No study has investigated this correlation in a flexibility program so far. The purpose of this study was to observe the influence of the range of motion (ROM) limitation on ROM gain after a six-week shoulder external rotation stretching training. 30 volunteer physiotherapy students, with limited shoulder external rotation ROM were randomly assigned to one of three groups: control group (CG), experimental group 1 (EG1), with wider ROM limitation, and experimental group $2(E G 2)$, with narrower ROM limitation. Subjects in the experimental groups were submitted to a 6-week program of active shoulder external rotation stretching. One-way ANOVA showed a significant difference in mean external rotation ROM gains among all three groups ( $\mathrm{p}=0.001)$, with $\mathrm{EG} 1$ having the highest ROM increase $\left(30.1^{\circ} \pm 8.6^{\circ}\right)$, followed by EG2 $\left(15.2^{\circ} \pm 7.5^{\circ}\right)$ and $C G\left(1.1^{\circ} \pm 5.8^{\circ}\right)$. The Pearson correlation analysis showed a significant negative correlation between previous ROM and ROM improvement ( $r=-0.70, p=0.001$ ). The results of our study showed that shoulder external rotation ROM limitation influences ROM improvement after a six-week stretching program in a healthy young population. Subjects with more pronounced ROM limitation respond with a more remarkable ROM improvement.
\end{abstract}

Keywords: muscle, rehabilitation, training

\section{INTRODUÇÃO}

A utilização de exercícios de alongamento tem se mostrado efetiva no aumento da amplitude de movimento (ADM) em diferentes articulações ${ }^{(1-10)}$. Clinicamente, estes exercícios são prescritos em condições de grande limitação de ADM, como em períodos pós-imobilização; assim como em pequenas limitações de ADM observadas em indivíduos saudáveis.

Segundo Kraemer e Hakkinen ${ }^{(11)}$, o potencial de alteração de uma variável física é maior quando se inicia um treinamento. Este fenômeno pode ser chamado de janela de adaptação. Sendo assim, quando menos treinado for o indivíduo, maior será seu potencial de alteração com o treinamento e maior será sua janela de adaptação. Ao contrário, esta deverá ser menor quando o indivíduo for mais treinado, ou se o mesmo já tiver alcançado um alto nível de condicionamento na variável física a ser desenvolvida.

O fenômeno da janela de adaptação tem sido comprovado em diferentes capacidades físicas. Alguns estudos observaram que indi- 
víduos não treinados, com menor capacidade aeróbica ou de força, apresentam melhora mais pronunciada destas variáveis com o treinamento ${ }^{(12,13)}$.

Apesar da idéia de que o ganho de ADM também seja influenciado pelo nível prévio de flexibilidade, nenhum estudo observou a influência da limitação da ADM sobre o ganho da mesma após um período de alongamento.

Sendo assim, os objetivos deste estudo foram: (1) comparar o ganho de ADM de rotação externa de ombro entre indivíduos com maior e menor limitação de ADM após seis semanas de alongamento e (2) observar uma possível correlação entre nível prévio de ADM de rotação externa de ombro e aumento da mesma após seis semanas de alongamento.

\section{MÉTODOS}

\section{Amostragem}

Participaram deste estudo 30 voluntários (14 homens e 16 muIheres), com media de idade de 21,3 \pm 2,7 anos, estudantes do curso de Fisioterapia da Pontifícia Universidade Católica de Minas Gerais. Todos os indivíduos apresentavam limitação da ADM de rotação externa de ombro (menos que $9^{\circ}$ ) quando mensurados em posição de adução de ombro (posição neutra) ${ }^{(14-16)}$. Além disto, os mesmos não apresentavam qualquer sinal de patologia no membro superior e coluna cervical. Aqueles sujeitos que praticassem algum esporte se comprometeram a manter o mesmo nível de treinamento durante o estudo. Este estudo foi aprovado pelo Comitê de Ética em Pesquisa da Pontifícia Universidade Católica de Minas Gerais. Todos os voluntários assinaram um termo de consentimento livre e esclarecido aceitando a participação no estudo.

\section{Medidas}

Para medida e treinamento da ADM, foi utilizado um instrumento denominado Cadex, construído pelo Laboratório de Biomecânica da Pontifícia Universidade Católica de Minas Gerais (Figura 1). Trata-se de uma cadeira adaptada com dois braços laterais (à esquerda e direita) que permitem a mensuração da ADM de rotação externa de ombro em posição neutra nos plano sagital e frontal, com o cotovelo flexionado a 90 graus e antebraço em neutro. Cada braço da cadeira é preso a uma barra vertical fixa na lateral do assento, possuindo um ajuste vertical que

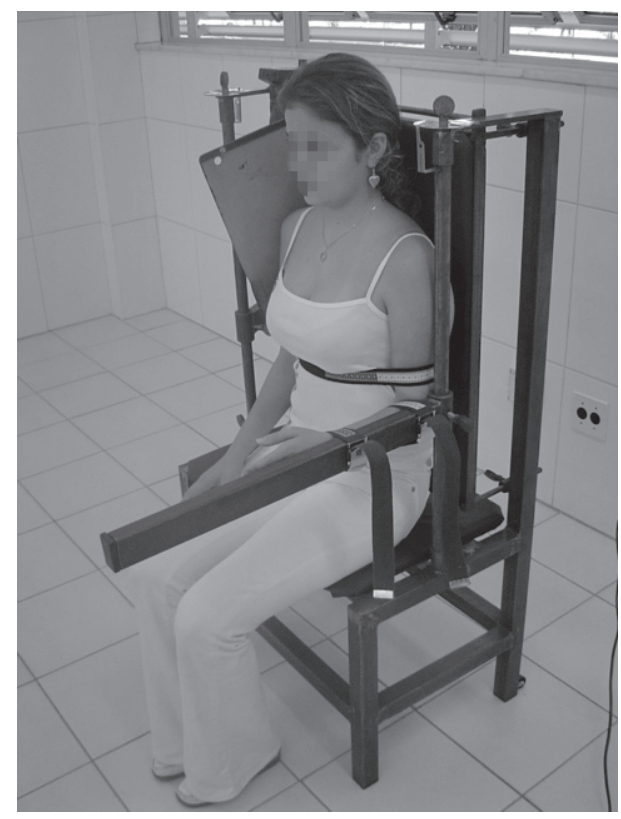

Figura 1. Voluntário na CADEX possibilita sua adaptação a indivíduos com diferentes comprimentos de braço. Uma vez que o braço da cadeira é movimentado no sentido da rotação, a barra vertical onde o mesmo é fixado, acompanha o movimento. Com a ajuda de um indicador posicionado na parte superior da barra, é feita a leitura da ADM alcançada em um transferidor, da marca Protactor $^{\oplus}$, fixo na parte superior da cadeira. A cadeira possui ainda uma regulagem da posição do encosto, permitindo assim adaptação a diferentes posicionamentos de escápulas e diferentes diâmetros ântero-posteriores de tronco, o que influenciaria a posição do ombro. Anterior ao encosto, há um anteparo para fixação lateral do tronco. Este anteparo pode ser ajustado lateralmente, sendo posicionado junto ao ombro contra-lateral a ser testado, o que permite a fixação da posição do voluntário. Para permitir o controle da velocidade do movimento de rotação externa, foi utilizado um metrônomo.

Em um estudo prévio, este instrumento apresentou boa confiabilidade intra- (ICC=.98) e inter- (ICC=.96) examinador após um período de 48 horas $^{(17)}$.

\section{Tratamento experimental}

Os indivíduos foram divididos de forma randomizada em dois grupos: grupo controle (GC, $N=10)$, e grupo experimental ( $G E, N=20$ ). Uma avaliação inicial da medida de ADM passiva de rotação externa de ombro (pré-teste) foi feita em todos os indivíduos. O lado do ombro a ser testado foi escolhido de forma aleatória.

Após esta avaliação, foi calculado a mediana dos valores de ADM do grupo GE. Este grupo foi então subdividido em dois grupos: grupo experimental 1 (GE1, N=10 ombros), com maior limitação de ADM (valores de ADM abaixo da mediana) e grupo experimental 2 (GE2, $\mathrm{N}=10$ ombros), com menor limitação de ADM (valores de ADM acima da mediana).

Os indivíduos dos dois grupos experimentais foram então submetidos a uma sessão de alongamento, duas vezes por semana, durante seis semanas, perfazendo um total de 12 sessões de alongamento. Cada sessão constou de uma série com uma repetição de 30 segundos de alongamento passivo estático para ganho de ADM de rotação externa de ombro. Este protocolo se mostrou suficiente para o ganho de ADM após um período de treinamento ${ }^{(2)}$. Todas as sessões de alongamento foram realizadas no mesmo instrumento no qual foram feitas as medidas de ADM (Cadex) e tiveram a supervisão de um examinador. Durante o alongamento, o voluntário deveria, atingir a ADM máxima de rotação externa tolerada e permanecer nesta posição por 30 segundos. O grupo controle permaneceu sem treinamento durante este período.

Após o período de treino, foi feita uma nova medida de ADM de rotação externa de ombro (pós-teste) em todos os grupos. Os avaliadores que participaram da medida da ADM e do treinamento eram cegos em relação aos grupos experimentais.

\section{Análise estatística:}

Para comparação das médias dos ganhos de ADM obtidos nos grupos GC, GE1 e GE2, foi utilizado um teste de análise de variância ANOVA one-way. Para localizar diferenças entre os grupos, foi utilizado um teste Post Hoc Tukey. Para observar uma possível correlação entre a ADM prévia e o ganho de ADM nos grupos experimentais, foi utilizado um teste de correlação de Pearson. Todos os testes utilizaram um nível de significância de 0,05.

\section{RESULTADOS}

Na comparação entre as médias dos ganhos de ADM de rotação externa de ombro após o período de treino, foram encontradas diferenças significativas entre todos os grupos ( $p=0,001)$. O grupo GE1, com maior limitação de ADM, apresentou ganho de ADM significativamente maior 
quando comparado ao grupo GE2 e GC. O grupo GE2, com menor limitação de ADM, apresentou ganho de ADM também significativamente maior, quando comparado ao GC ( $p=0,001)$ (Tabela 1).

No teste de correlação de Pearson, a ADM prévia apresentou correlação negativa com o ganho de $A D M(r=-0,70, p=0,001)$, ou seja, quanto menor a ADM prévia de rotação externa de ombro, maior o ganho de ADM.

Tabela 1. Média e desvio padrão das ADMs de rotação externa de ombro obtidas no pré, pós-teste, ganho e $\Delta \%$

\begin{tabular}{l|c|c|c}
\hline & GC (N=10) & GE1 (N=10) & GE2 (N=10) \\
\hline Pre-teste $(\circ)$ & $71,3 \pm 12,8$ & $63,4 \pm 7,8$ & $80,0 \pm 6,7$ \\
\hline Pós-teste $\left(^{\circ}\right)$ & $72,3 \pm 13,2$ & $93,4 \pm 9,1$ & $95,2 \pm 8,2$ \\
\hline Ganho $\left(^{\circ}\right)$ & $1,1 \pm 5,8^{*}$ & $30,1 \pm 8,6^{*}$ & $15,2 \pm 7,5^{*}$ \\
\hline$\Delta \%$ & 1,4 & 47,3 & 19,0 \\
\hline
\end{tabular}

* $d f=29, F=35,7, p=0,001$

\section{DISCUSSÃO}

Este estudo observou que indivíduos com maior limitação de ADM de rotação externa de ombro obtiveram maior ganho de ADM após um programa de alongamento de seis semanas, quando comparados a indivíduos com menor limitação de ADM. Após o período de treino, o GE1 apresentou quase o dobro do ganho de ADM quando comparado ao grupo GE2. Quando observados os valores de $\Delta \%$, esta diferença foi ainda maior (GE1=47,3\%, GE2=19,0\%). Uma correlação negativa entre nível prévio de ADM e ganho da mesma foi também encontrada.

Outros estudos têm demonstrado esta tendência em relação a outras variáveis físicas. Ahtiainen et al. ${ }^{(12)}$ observaram que atletas destreinados apresentaram ganho de força de 20,9\% contra apenas 3,9\% de ganho observados em atletas treinados, após um período de 21 semanas. Gimenez et al.(13) mostraram que indivíduos destreinados aumentaram significativamente mais seu VO2Max quando comparados a indivíduos treinados. (29,9\% e 6,6\% respectivamente). Os resultados deste estudo sugerem que a flexibilidade tem comportamento similar a outras capacidades físicas como força e resistência. No nosso conhe- cimento, nenhum estudo observou este padrão de alteração em um treino de flexibilidade.

Algumas hipóteses, como alterações estruturais ${ }^{(18,19)}$ e aumento da tolerância a dor ${ }^{(20-22)}$ poderiam explicar o aumento de ADM observado neste estudo. Os mecanismos que explicam a correlação entre limitação de ADM e potencial de aumento da mesma são desconhecidos. Porém, parece que um ou ambos os mecanismos: modificação da tolerância ao alongamento e alterações estruturais tende a ocorrer de forma mais rápida ou intensa quando a ADM se encontra mais limitada.

A utilização da medida de ADM e da técnica de alongamento de forma passiva, ou seja, controlada pelo examinador, pode ser considerada uma limitação deste estudo. A escolha deste tipo de técnica foi feita para possibilitar o controle da velocidade do movimento. Para garantir que todas as medidas fossem feitas na mesma velocidade, o examinador deveria visualizar o transferidor preso na parte superior da cadeira e com ajuda de um metrônomo, controlar a velocidade do movimento. Porém, a interrupção do movimento era sempre determinada pelo voluntário, que ao perceber a sensação de desconforto máximo tolerado, solicitava a parada do mesmo. Além disto, os examinadores eram cegos em relação aos grupos experimentais. Sendo assim, não acreditamos que este fator possa ter influenciado de forma significativa os nossos resultados.

Os achados deste estudo são limitados à população estudada, portanto, não devem ser extrapolados para outras articulações ou movimentos, ou ainda para indivíduos com alguma patologia.

\section{CONCLUSÃO}

Os resultados deste estudo mostraram que indivíduos jovens e saudáveis, com maior limitação de ADM de rotação externa de ombro, conseguiram maior ganho de ADM, quando comparados a indivíduos com menor limitação desta ADM, após um programa de alongamento estático de seis semanas. Além disto foi observada uma correlação entre a limitação de ADM e o potencial aumento desta. Quanto maior a limitação de ADM, maior o ganho de ADM. Mais estudos são necessários para investigar os mecanismos relacionados a esta correlação.

Todos os autores declararam não haver qualquer potencial conflito de interesses referente a este artigo.

\section{REFERÊNCIAS BIBLIOGRÁFICAS}

1. Bandy WD, Irion JM. The effect of time on static stretch on the flexibility of the hamstring muscles. Phys Ther. 1994; 74: 845-52

2. Bandy WD, Irion JM, Briggler M. The effect of time and frequency of static stretching on flexibility of the hamstring muscles. Phys Ther. 1997; 77: 1090-6.

3. Bandy WD, Irion JM, Briggler $M$. The effect of static stretch and dynamic range of motion training on the flexibility of the hamstring muscles. J Orthop Sports Phys Ther. 1998; 27: 295-300.

4. Borms J, Van Roy P, Santens JP, Haentjens A. Optimal duration of static stretching exercises for improvement of coxo-femoral flexibility. J Sports Sci. 1987; 5: 39-47.

5. Chan $S P$, Hong $Y$, Robinson PD. Flexibility and passive resistance of the hamstrings of young adults using two different static stretching protocols. Scand J Med Sci Sports. 2001; 11: 81-6.

6. Cipriani D, Abel B, Pirrwitz D. A comparison of two stretching protocols on hip range of motion: implications for total daily stretch duration. J Strength Cond Res. 2003; 17: 274-8.

7. Feland JB, Marin HN. Effect of submaximal contraction intensity in contract-relax proprioceptive neuromuscular facilitation stretching. Br J Sports Med. 2004; 38: E18.

8. Feland JB, Myrer JW, Schulthies SS, Fellingham GW, Measom GW. The effect of duration of stretching of the hamstring muscle group for increasing range of motion in people aged 65 years or older. Phys Ther. 2001; 81:1110-7.

9. Winters MV, Blake CG, Trost JS, et al. Passive versus active stretching of hip flexor muscles in subjects with limited hip extension: a randomized clinical trial. Phys Ther. 2004; 84: 800-7.

10. Youdas JW, Krause DA, Egan KS, Therneau TM, Laskowski ER. The effect of static stretching of the calf muscle-tendon unit on active ankle dorsiflexion range of motion. J Orthop Sports Phys Ther. 2003; 33: 408-17.

11. Kraemer WJ, Hakkinen K. Strength Training for Sport 1ed Oxford: Blackwell Publishing; 2002.
12. Ahtiainen JP, Pakarinen A, Alen M, Kraemer WJ, Hakkinen K. Muscle hypertrophy, hormonal adaptations and strength development during strength training in strength-trained and untrained men. Eur J Appl Physiol. 2003; 89: 555-63.

13. Gimenez M, Cereceda V, Teculescu D, Aug F, Laxenaire MC. Square-wave endurance exercise test (SWEET) for training and assessment in trained and untrained subjects. III. Effect on VO2 max and maximal ventilation. Eur J Appl Physiol Occup Physiol. 1982; 49: 379-87.

14. Andrews JR, Wilk KE. The athlete's shoulder New York: Churchill Livingstone; 1994.

15. Magee DJ. Orthopedic Physical Assessment. 3rd ed Philadelphia: W.B. Saunders; 1997.

16. Schenkman M, Rugo de Cartaya V. Kinesiology of the shoulder complex. J Orthop Sports Phys Ther. 1987; 8: 438-450.

17. Azevedo DC. Confiabilidade intra e interexaminador de um instrumento para medida e treinamento da ADM de rotação externa de ombro. Revista Brasileira de Medicina do Esporte. 2004; 10: 434.

18. De Deyne PG. Application of passive stretch and its implications for muscle fibers. Phys Ther. 2001; 81:819-27

19. Reid DA, McNair PJ. Passive force, angle, and stiffness changes after stretching of hamstring muscles. Med Sci Sports Exerc. 2004; 36: 1944-8.

20. Halbertsma JP, Goeken LN. Stretching exercises: effect on passive extensibility and stiffness in short hamstrings of healthy subjects. Arch Phys Med Rehabil. 1994; 75: 976-81.

21. Halbertsma JP, van Bolhuis Al, Goeken LN. Sport stretching: effect on passive muscle stiffness of short hamstrings. Arch Phys Med Rehabil. 1996; 77: 688-92.

22. Magnusson SP, Simonsen EB, Aagaard P, Sorensen H, Kjaer M. A mechanism for altered flexibility in human skeletal muscle. J Physiol. 1996; 497: 291-8. 